\title{
Design of Partial Discharge Detection System for XLPE Cable Based on Zigbee Technology
}

\author{
S.B.HUANG ${ }^{1}$, J.B.WANG ${ }^{1}$, S.H.HE ${ }^{1}$ \& Q.H.ZHAN ${ }^{1}$ \&Y.M.ZHANG ${ }^{2}$ \& Y.P.XU ${ }^{2}$ \\ ${ }^{1}$ Guangdong Power Grid Corporation Foshan Power Supply Bureau, Foshan, China \\ ${ }^{2}$ Shanghai Jiao Tong University, Shanghai, China
}

KEYWORD: ZigBee; power cable; partial discharge; on-line monitoring; wireless communication ABSTRACT: The Internet of Things (IOT) oriented design of cable partial discharge detection system was proposed. In the novel system, the IOT module supporting ZigBee protocol is adopted and the Z-stack protocol stack is optimized. The system can on-line monitor partial discharge of 30 cables. Experimental results indicate that this novel system is with high reliability and easy for installation and extension.

\section{Introduction}

Due to convenience, operation and maintenance simplicity, good heat insulation properties and other characteristics, power cable are widely used in transmission and distribution network ${ }^{[1-4]}$. Present mostly used power cable is laid in a dozen or even a few decades ago. Due to the lack of effective means to judge the safety of the cable, it can only replace the cable or cable accessory after a failure. Power operation happens when replacement not only has a great impact on industrial production, but also seriously disturbed the normal life of the public. Therefore, research and development of a fast multiple powers cable safety hazards detection and discovery system has a very important practical significance.

The cable partial discharge on-line monitoring system currently used is wired (such as fiber) transmission mode of the PD signal. The system can only work in a fixed position and equipment installation is complex, difficult to move and has poor flexibility. It is difficult to full and dynamically cover monitoring cable running safety problems ${ }^{[5-9]}$. This paper presents a solution based on ZigBee wireless transmission technology. ZigBee technology is a wireless communication technology for short range, low data transmission rate ${ }^{[10]}$. A wireless communication network can be quickly built up to on-line monitor multi-channel cable PD signal. It is a new scheme of power cable partial discharge monitoring system.

\section{ZIGBEE WIRELESS COMMUNICATION TECHNOLOGY}

In recent years, wireless local area network WPAN technology strives to develop a variety of standard techniques and ZigBee emerged. It is a new short-range, low power, low cost, low complexity of wireless network technology, taking IEEE 802.15.4 MAC layer and physical layer, and increasing the logical network, standardized network security and application layer.

Unlike some other communication technologies, ZigBee do not pursue high-speed, long-distance but for specific application requirements, locking only at a rate of tens of $\mathrm{kps}$, a few meters to tens of meters - the ability to achieve wireless group network communication. Thus ZigBee advantage is not that it's the technology itself, but the key is rich and convenient application. In particular, in the industrial field, the use of sensors and ZigBee networks, making automatic data acquisition analysis, and processing easier, can be an important part of the decision support system.

\section{ZIGBEE BASED CABLE PD MONITORING SYSTEM}

\section{Cable PD monitoring system overall architecture}

Cable partial discharge monitoring system mainly consists of high-frequency sensors, signal amplification module, intelligent detection front-end, wireless communication module to detect and analyze diagnostic center terminal, the overall structure of the monitoring system is shown in Fig. 1. 
High-frequency transducer extracted PD signal after pre-amplifier is transferred to the front intelligent detection, each detection front-end can support 8-way cable PD signal acquisition, intelligent front-end detection signal preprocessing, the extracted signal characteristics parameters, the wireless network will be sent to the characteristic parameter detecting terminal, and then realize there is analysis and diagnosis center analysis and diagnosis of PD signal cable.

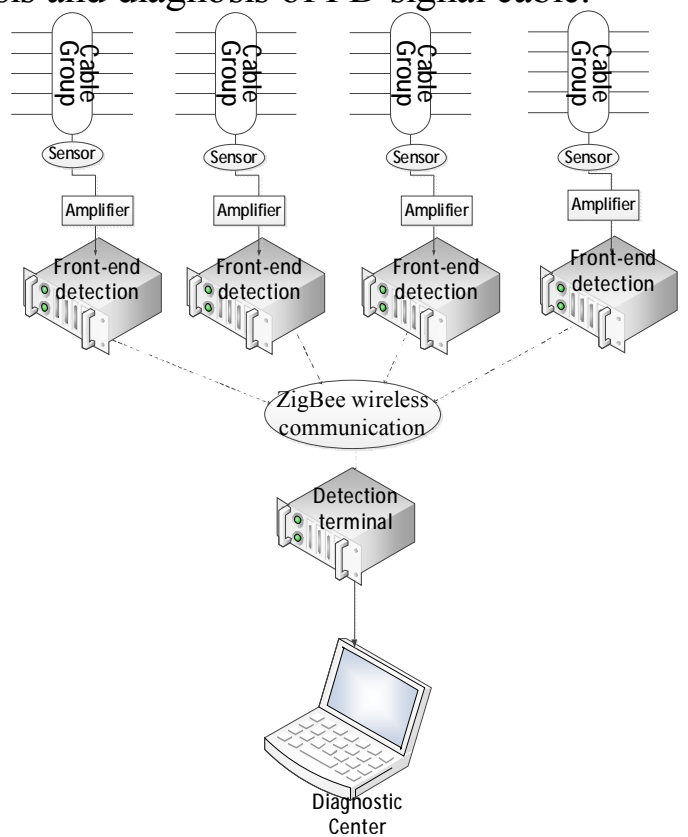

Fig. 1 System structure

\section{Data transmission node}

Data transmission node is connected to the front end intelligent detection signal acquisition module and its position is relatively fixed. Data transmission node receives data a, processes data and sends out the wireless network. This paper designed cable PD online monitoring system has four data transmission node, together with the data acquisition module is mounted inside the front intelligent detection, data transmission node XBee module as a Router.

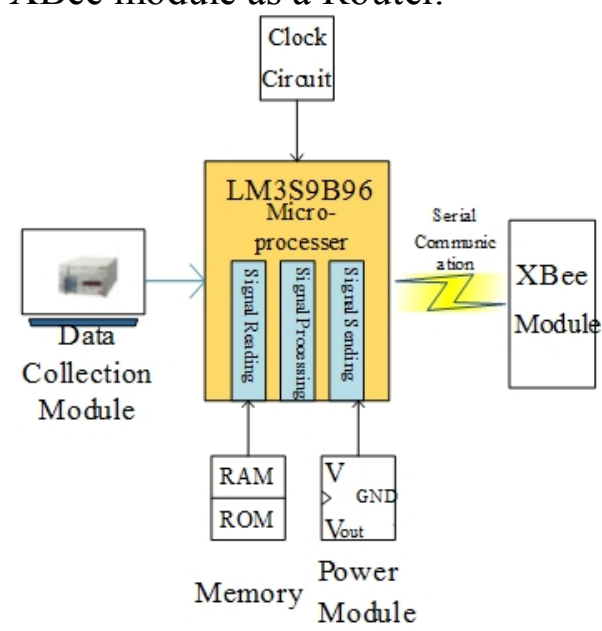

Fig. 2 Data transmission node configuration diagram

The internal structure of the data transmission node is shown in Fig. 2. TI's processor LM3S9B96 microcontroller is selected. DiGi's selection of ZigBee module XBee wireless communication module, complete with a receiving device communications and data transmission.

\section{Data receiving node}

Data receiving node main function is to receive the information on the power cable PD signal corresponding treatment based on XBee module. This section includes a message receiving node, which XBee module as Coordinator, the structure shown in Fig.3. At the same time, the node is connected 
to the diagnostic server, send the received information diagnostic server, to analyze and diagnose cable PD signal.

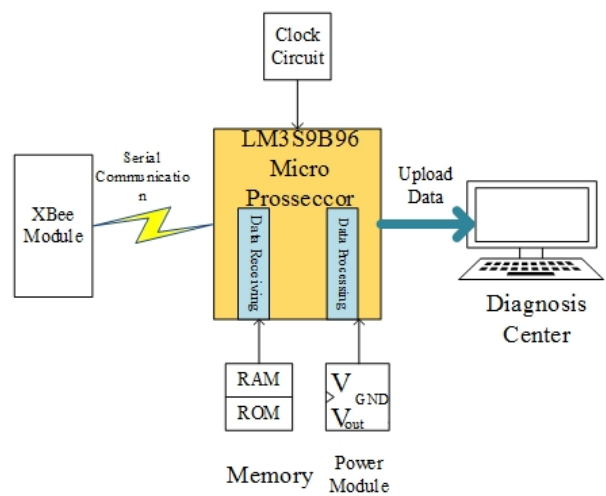

Fig.3 data receving node configuration diagram

\section{POWER CABLE PD MONITORING SYSTEM SOFTWARE DESIGN}

\section{The cable PD signal acquisition module}

Power cables partial discharge monitoring system to detect intelligent front-end capture card is mainly used for collecting sensor detected discharge signal. Acquisition card and data transmission node connected to the microprocessor in the LM3S9B96, data collection program flow chart shown in Fig.4.

LM3S9B96 by writing to the program read the acquisition card to the PD data and stored as a matrix of $50 * 60.3 .2$ And 3.3 need to be modified to use the measured partial discharge waveform samples do for data transmission verification system reliability.

\section{Peer communication}

We select two XBee nodes communication transceiver to do single point matrix data experiment. One node is Coordinator, another node for the Router. The distance of two-node is about $10 \mathrm{~m}$. By writing to the sender to make a XBee Router node, node sends a $60 * 50$ matrix data. Every time sending 50 lines data and transmission interval is as $1 \mathrm{~ms}$.

Router's X-CTU terminal interface shows the received data and Coordinator's X-CTU terminal interface shows returned instructions after Router received. Which transmit data and receive data Router consistent Coordinator, Coordinator module receives a return instruction is 50 times $7 \mathrm{E} 00$ 07 8B 0116 C8 000000 95, show 50 times the data were sent successfully. 3.2 And 3.3 need to be modified to use the measured partial discharge waveform samples to do for data transmission verification system reliability. 


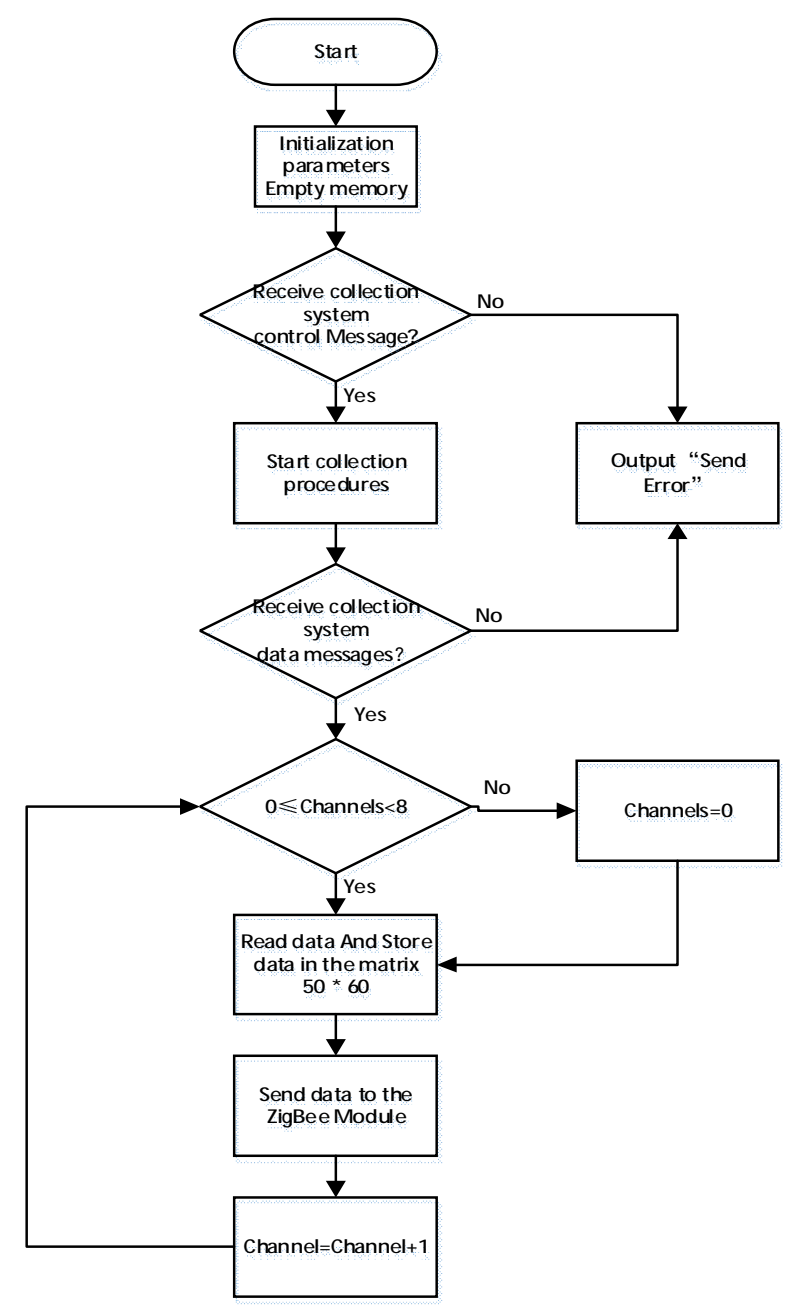

Fig.4 Data acquisition program flow

Write a receiving program, the program flow chart shown in Figures 5. You will load the receiving program to the Coordinator node LM3S9B96, making Router received all the data again reduced to $60 \times 50$ matrix memory. 


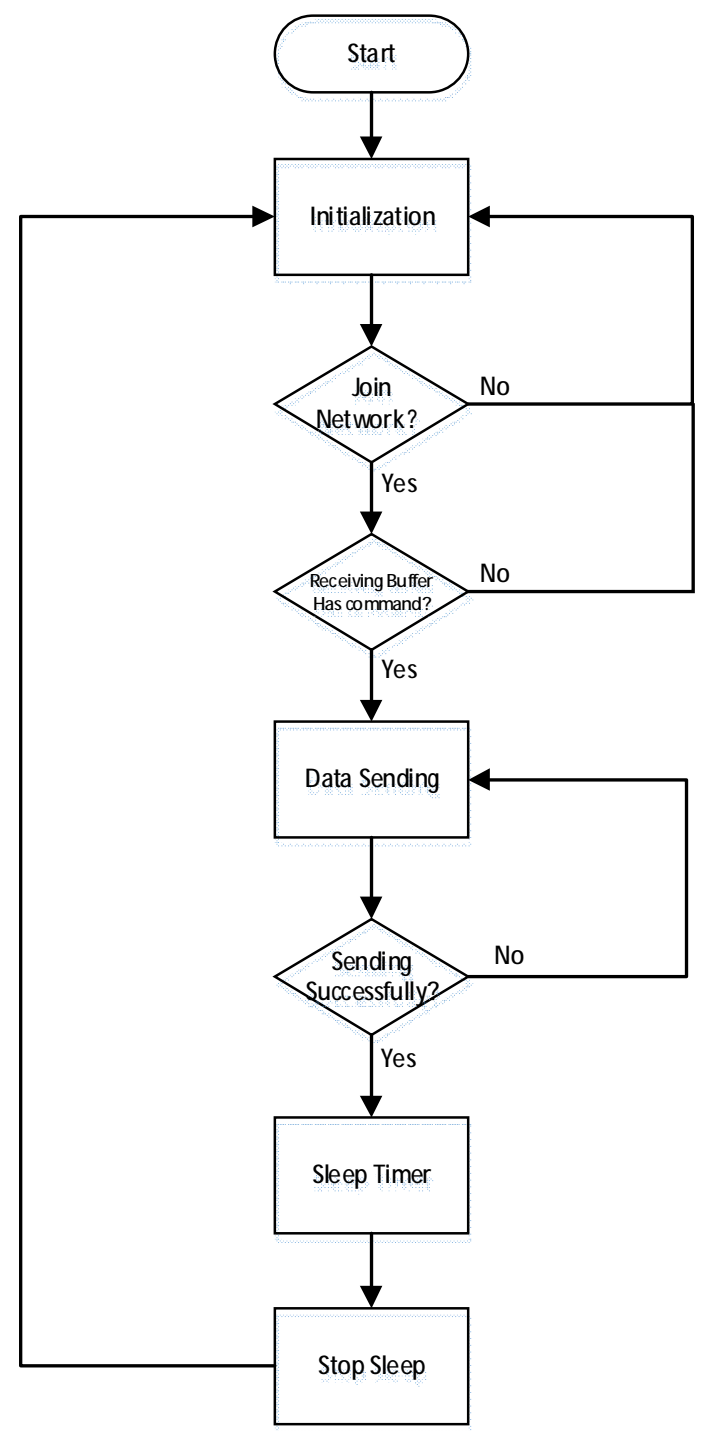

Fig.5 Router node program flow

\section{Field Application Examples}

We select two XBee nodes communication transceiver to do single point matrix data experiment. One node is Coordinator, another node for the Router. The distance of two-node is about 10m. By writing to the sender to make a XBee Router node, node sends a $60 * 50$ matrix data. Every time sending 50 lines data and transmission interval is as $1 \mathrm{~ms}$. 


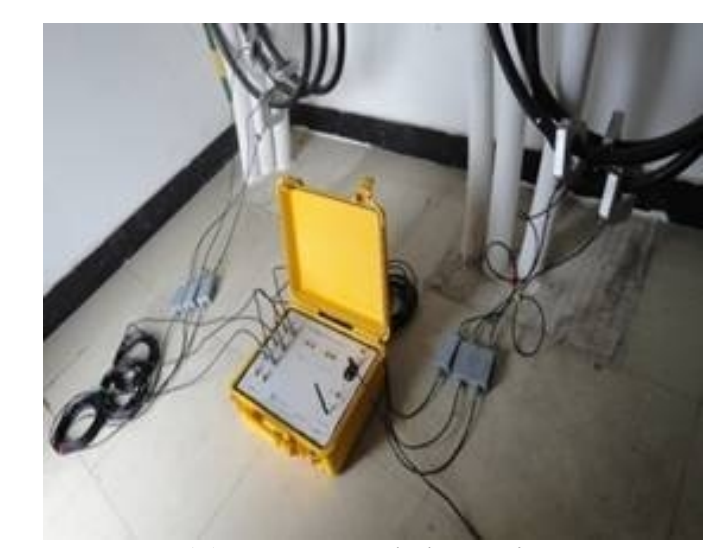

(a) Front-end detection

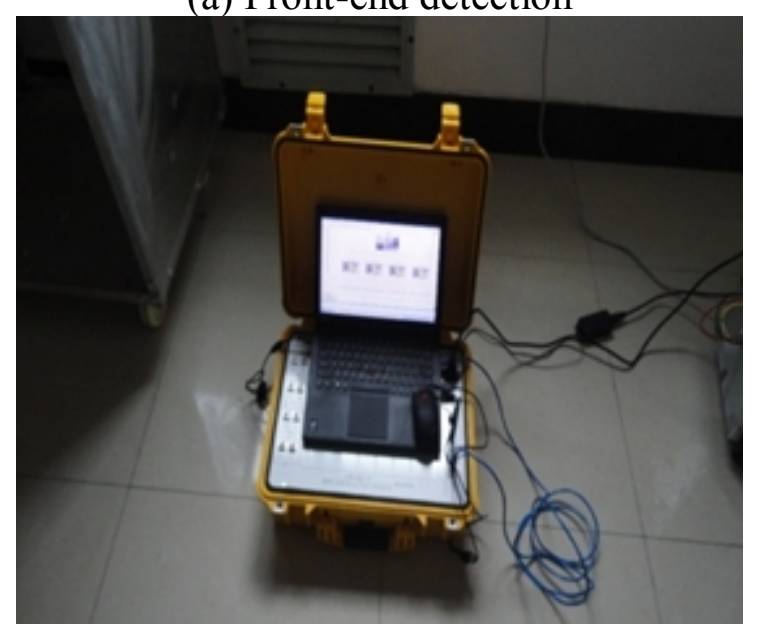

(b) Detecting terminal

Fig.6 ZigBee-based cable on-line monitoring system

Developed based on ZigBee technology cable on-line monitoring system is shown in Fig.6, comprising a plurality of detecting the front and a test terminal. After the detection of the front get cable PD signal preprocessing, signal characteristic parameters are extracted, via ZigBee wireless network will be sent to the characteristic parameter detecting terminal, and then through the analysis and diagnosis center for cable PD signal analysis and diagnosis.

The new cable partial discharge monitoring system installation and operation in a number of substations, cable lines for dozens of PD are continuous on-line monitoring.

\section{CONCLUSION}

This paper studies the ZigBee wireless transmission technology and its application in cable PD online monitoring and designs cable PD on-line monitoring system based on ZigBee wireless transmission technology.

Compared with existing cable PD on-line monitoring system, this system has characteristics as follows:

1) Simple system components to reduce the complexity of the cable, which can effectively improve the efficiency of field work;

2) System structure is flexible, scalable, based on the requirements of the test device, add or delete monitoring front and channel;

3 ) Low system cost and high reliability, the system front-end processing, to the rear end of the transmission characteristic parameters, which greatly improves the communication efficiency, enhance system reliability.

\section{ACKNOWLEDGMENT}

This work was supported by the Guangdong Power Grid Corporation (K-GD2013-047), The research project of field acceptance and operation maintenance's tests and detection methods of medium voltage cable. 


\section{REFERENCES}

[1] YANG Li-ming, ZHU Zhi-en, YANG Rong-kai, et al.Insulation Material and Structure Design of HVDC Flexible Cables[J]. Automation of Electric Power System ,2013,37(15):117-124.

[2] HUO Zhen-xing. Research on Insulation Degradation of $10 \mathrm{kV}$ XLPE Power Cables[D]. Tianjing University, 2009. DOI:10.7666/d.y1676631.

[3] CIGRE Working Group. After laying tests on high voltage extruded insulation cable system [J]. Electra, 1997( 173): 33-41.

[4] ZHANG Z S, XIAO D M, LI Y. Rogowski air coil sensor technique for on-line partial discharge measurement of power cables [J]. IET Science, Measurement \& Technology, 2009, 3( 3):187-196.

[5] LI Wen-quan,LAN Sheng.Review of Common On-line Monitoring Methods for Power Cable Conditions[J].Electric Switchgear,2013(6): 1-7.

[6] LI Yong-liang, ZHANG Tao, LUO Hong-li, et al.Application of Wireless Private Network in Ningxia $330 \mathrm{kV}$ Transmission Line Monitoring [J]. Electrical Automation, 2014, 36(2):39-42.

[7] ZHAO Zhong-yuan, LU Zheng-yu, DUAN Nai-xin, et al. Research and Development of Online Monitoring System with Partial Discharge in XLPE Power Cables[J]. Automation of Electric Power System,2004,28(5): 59-62.

[8] CHEN Rui-long, QIAN Yong, YE Hai-feng, et al.The Development of an On-line Monitoring System Adopting Partial Discharge of XLPE Cables [J]. Electrical Automation , 2014, 36(2) :101103.

[9] ZHU Ying-wei, ZHOU Kai, YOU Shi-yu, et al.Design and Experiment of Partial Discharge Detector Based on Ultrasonic Wave for Cable Accessories [J]. Electric Wire \& Cable,2013 (2):33-36.

[10] CHEN Zhen-hua, CHEN Xiao-yan, LIU Xing-yi.A Temperature Monitoring System for Warm Water Discharge from Coastal Power Plant Based on Zigbee and GPRS Networking [J]. Computer Measurement \& Control, 2013, 21(9): 2400-2402. 